\title{
Educação em saúde no autocuidado contra o câncer de mama
}

\author{
Health Education in self-care against breast cancer
}

\author{
Diego Augusto Lopes Oliveira'
}

\section{RESUMO}

Estudo de revisão integrativa com objetivo de conhecer como as ações de educação em saúde influenciam o autocuidado durante $o$ tratamento quimioterápico para câncer de mama.A pesquisa foi estruturada em seis etapas e buscou responder a seguinte questão norteadora: Como as atividades de educação em saúde interferem no autocuidado da mulher com câncer de mama em quimioterapia? Foram utilizados dados primários em bases de dados de livre acesso com critérios estabelecidos para busca em periódicos nos idiomas português, inglês e espanhol através das modalidades de pesquisa e níveis de evidência no período de 2000-2017. Nas publicações analisadas, todas publicadas em língua inglesa, observouse que o papel do enfermeiro na educação em saúde e as estratégias adotadas promovem fortalecimento de ações de autocuidado e autogerenciamento das reações adversas pela paciente e possibilitam melhoras na qualidade de vida pós quimioterapia. Torna-se importante o investimento em ações educativas à mulheres nesta modalidade de tratamento por permitir maior impacto na prevenção de complicações oriundas das reações adversas, potencialização do autocuidado e redução dos custos com tratamentos adicionais.

Descritores: Neoplasia das mamas; Quimioterapia; Educação em Saúde;Autocuidado.

\begin{abstract}
Integrative review study aiming to understand how health education actions influence self-care during chemotherapy treatment for breast cancer. The research was structured in six stages and sought to answer the following guiding question: How do health education activities interfere with the self-care of women with breast cancer in chemotherapy? We used primary data in free access databases with established criteria for searching in journals in Portuguese, English and Spanish through research modalities and levels of evidence in the period 2000-20 17. In your. In the analyzed publications, all published in English, it was observed that the role of nurses in health education and the strategies adopted promote self-care actions and self-management of adverse reactions by the patient and enable improvements in quality of life after chemotherapy. It is important to invest in educational actions for women in this treatment modality because it allows greater impact in the prevention of complications resulting from adverse reactions, self-care enhancement and reduction of costs with additional treatments.
\end{abstract}

Descriptors: Breast Neoplasms; Chemoterapy; Health Education; Selfcare. 


\section{INTRODUÇÃO}

O câncer de mama é um grupo heterogêneo de doenças, com comportamentos distintos.A heterogeneidade deste câncer pode ser observada pelas variadas manifestações clínicas e morfológicas, diferentes assinaturas genéticas e consequentes diferenças nas respostas terapêuticas'.

A incidência desse tipo de tumor em mulheres cresce progressivamente a partir dos 40 anos e, no Brasil, estimam-se 59.700 casos novos de câncer de mama, para cada ano do biênio 2018-2019, com um risco estimado de 56,33 casos a cada 100 mil mulheres ${ }^{2}$. Diante deste panorama é feito, nos últimos anos, o investimento em ações pautadas na promoção de medidas para rastreamento e diagnóstico precoce da doença que aumentam o acesso aos métodos de avaliação da mulher e possibilitam maior intervenção dos profissionais de saúde no objetivo de ofertar tratamento o mais brevemente possível ${ }^{3}$.

Com a confirmação da doença, a mulher com câncer mamário passa por tratamento que comumente é composto por cirurgia, quimioterapia, radioterapia, hormonioterapia e imunoterapia, que podem ser indicadas isoladamente ou combinadas entre $\mathrm{si}^{4}$. A Quimioterapia (QT) apresenta alta utilização sendo ajustado ao estadiamento tumoral e a indicação de associação a outras terapias, sendo que sua utilização diminui as chances de recidiva, o tratamento de metástases não detectáveis e possibilita maior sobrevida da mulher ${ }^{5}$.

Rotineiramente essa modalidade de tratamento traz inúmeros efeitos colaterais, os quais relacionam o fato de não afetarem exclusivamente as células tumorais ${ }^{6}$. Os efeitos mais frequentes entre mulheres com câncer mamário em quimioterapia são de origem hematológica, gastrointestinais e relacionados à cardiotoxicidade, hepatotoxicidade, toxicidade pulmonar, neurotoxicidade, disfunção reprodutiva, toxicidade vesical e renal, alterações metabólicas, toxicidade dermatológicas e reações alérgicas e anafilaxia ${ }^{7}$.

Para manejo desses efeitos faz-se necessário a definição de um planejamento terapêutico individual pela Enfermagem e demais profissionais que prime pelas ações de educação em saúde a fim de reduzir os efeitos indesejados e potencializar o autocuidado. Uma vez orientada a mulher se torna participante ativa do tratamento e aprende a desenvolver estratégias individuais para redução dos efeitos com menos ansiedade e reduzindo possíveis complicações de saúde ${ }^{8}$.

Nesta perspectiva, compete ao enfermeiro e à equipe de saúde agir de forma acurada e conjunta para dar conta de demandas, tanto preventivas quanto de promoção da saúde e curativas. Assim, é importante que haja aproximação entre a equipe e usuários dos serviços de saúde, na medida em que esta permite uma difusão do conhecimento técnico-científico, unindo-os e desmistifi- cando a doença e tratamento, pois, quanto mais próxima da população, maior a possibilidade de viabilizar a identificação de fatores de risco e a busca pela prevenção e diagnóstico de complicações?.

Este estudo tem como objetivo conhecer como as ações de educação em saúde atuam sobre o autocuidado durante o tratamento quimioterápico para câncer de mama.

\section{METODOLOGIA}

Estudo do tipo revisão integrativa da literatura, que se trata de um método que proporciona a síntese de conhecimento e a incorporação da aplicabilidade de resultados de estudos significativos na prática ${ }^{10}$. Este método tem como principal finalidade reunir e sintetizar os estudos realizados sobre um determinado assunto, construindo uma conclusão, a partir dos resultados evidenciados em cada estudo, mas que investiguem problemas idênticos ou similares. Os estudos incluídos na revisão são analisados de forma sistemática em relação aos seus objetivos, materiais e métodos, permitindo que o leitor analise $\circ$ conhecimento pré-existente sobre o tema investigado".

A pesquisa foi norteada a partir da pergunta condutora:"Como as atividades de educação em saúde interferem no autocuidado da mulher com câncer de mama em quimioterapia?". Dando seguimento elegeu-se como ambiente de pesquisa as Bases de dados da LILACS (Literatura Latino-americana e do Caribe em Ciências da Saúde), MEDLINE (Medical Literature Analysis and Retrieval System Online) e BDENF (Base de dados em Enfermagem). As buscas dos periódicos foram norteadas pelos seguintes critérios de elegibilidade: Foram incluídos Artigos publicados no período de 2000 à 2017; Publicados nos idiomas: Português, Inglês e Espanhol; Com livre acesso nas bases de dados elencadas e rastreados como produto da busca dos descritores controlados das ciências da Saúde (DECS-BIREME): Câncer de Mama/Breast Neoplasms/ Neoplasias de la Mama, quimioterapia/chemoterapy/quimioterapia; educação em saúde/health education/Educacíon en Salud e Autocuidado/Selfcare/ Autocuidado utilizando o conector booleano "and" para cruzamentos dos descritores. Foram excluídas produções repetidas, trabaIho de conclusão de curso e residência, teses, dissertações, editoriais, resumos, carta de opinião e revisões de literatura, resumos de anais de congressos, boletins informativos e arquivos base de programas ou estratégias de intervenção popular nessa modalidade assistencial.

Seguindo Modelo da revisão" o desenvolvimento do estudo percorreu o seguinte caminho metodológico: $\mathrm{Na}$ primeira etapa foi estabelecida questão de pesquisa e elencados critérios para sua efetivação, nesse sentido, foi instrumentada a segunda etapa onde foram selecionados os artigos, sendo estes numerados em ordem crescente, de acordo com a sua ordem de aparição nas 
bases de dados relacionadas às publicações na área das ciências da saúde. $\mathrm{Na}$ terceira etapa foi realizada leitura dos resumos dos estudos a fim de compreender sua relação com o objetivo deste estudo e pergunta norteadora e permitir sua categorização utilizando o instrumento validado ${ }^{10}$ como meio de padronizar dados mais relevantes na realização dos estudos de revisão. Os estudos que não se enquadraram foram eliminados da composição amostral desta revisão. $\mathrm{Na}$ quarta fase/ etapa foi desenvolvida análise crítica dos estudos identificados como componentes da amostra do estudo. Nesta fase é verificado de forma crítica a sua relação com critérios relacionados a sua qualidade metodológica, importância e representatividade das informações ora mencionadas bem como da autenticidade dos dados discutidos. Após a etapa de categorização e leitura crítica dos estudos na quinta etapa foi reunido o produto da análise crítica e instrumentada a interpretação dos dados de impacto para o estudo, interligando estes ao produto teórico desenvolvido nas ciências da saúde ${ }^{12}$ para classificação dos estudos por nível de evidência a saber: Nível I: as evidências são provenientes de revisão sistemática ou metanálise de todos os relevantes ensaios clínicos randomizados controlados ou oriundos de diretrizes clínicas baseadas em revisões sistemáticas de ensaios clínicos randomizados controlados; Nível II: evidências derivadas de, pelo menos, um ensaio clíni- co randomizado controlado bem delineado; Nível III: evidências obtidas de ensaios clínicos bem delineados sem randomização; Nível IV: evidências provenientes de estudos de coorte e de caso controle bem delineados; Nível V: evidências originárias de revisão sistemática de estudos descritivos e qualitativos; Nível Vl: evidências derivadas de um único estudo descritivo ou qualitativo; e Nível VII: evidências oriundas de opinião de autoridades e/ou relatório de comitês de especialistas ${ }^{13}$

$\mathrm{Na}$ última etapa expressamos o resultado da busca e análise forma a evidenciar as contribuições desta para melhor entendimento do processo junto a prática e estabelecer, a partir desse apanhado de informações, medidas para melhoria no desenvolvimento dessas ações.

\section{RESULTADOS}

A pesquisa nas bases de dados elencadas foi realizada nos meses de fevereiro e março de 2018 e na figura I está destacada o desenvolvimento do processo delineado para tratamento da amostra gerada a partir da aplicação dos critérios de elegibilidade propostos para pesquisa. A técnica gerou o contingente de 08 artigos para aplicação das etapas de apreciação e, após o desenvolvimento destas, permitiu a análise crítica de 07 estudos. $O$ processo final de seleção, desenvolvido a partir da análise dos resumos das publicações fomentou a constituição de amostra composta por 03 publicações.

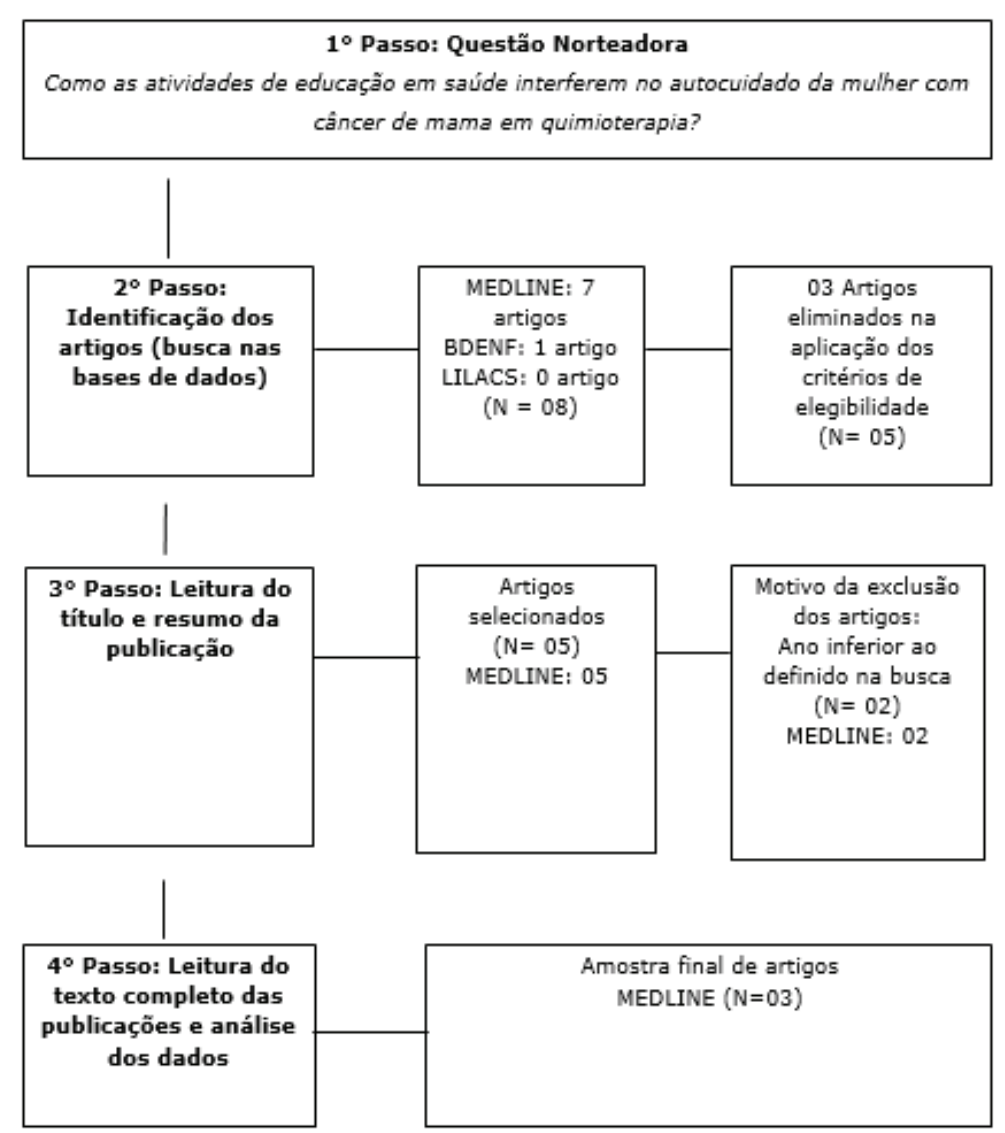

FIGURA 1 - Fluxograma de tratamento dos artigos buscados nas bases de dados, 2018 
Dos artigos analisados, observou-se que $100 \%(n=3)$ foram publicados em periódicos internacionais e em língua inglesa, sendo escasso publicações nos idiomas português e espanhol. Contatou-se também, que as publicações não apresentaram ano de publicação que oferecesse marco teórico para seu desenvolvimento e, em sua totalidade apresentam metodologia quantitativa. No tocante a definição do nível de evidência um dos manuscritos foi classificado em nível VI e os demais em nível IV.

Sendo assim, as informações dos estudos seleciona- dos foram estruturadas a partir dos dados do instrumento aplicado: título da publicação, autores, ano e revista, conforme o quadro I. Enquanto, o outro instrumento utilizado estruturado contemplou: objetivo, modalidade da pesquisa, nível de evidência e síntese dos resultados, conforme o quadro 2.

\section{DISCUSSÃO}

A morbidade por câncer de mama aumenta ao passar dos anos e a busca por tratamento é a cada dia mais difun-

QUADRO 1 - Síntese dos estudos sobre educação em saúde e quimioterapia contra câncer de mama, 2018

\begin{tabular}{|c|c|c|c|c|}
\hline ID & TÍTULO DA PUBLICAÇÃO & AUTORES & ANO & REVISTA \\
\hline 01 & $\begin{array}{l}\text { Self management Pilot study on women with breast cancer: } \\
\text { Lesson Learnt in Malaysia }{ }^{14} \text {. }\end{array}$ & $\begin{array}{l}\text { SY Loh } \\
\text { CH Yip } \\
\text { T Packer } \\
\text { KF Quek }\end{array}$ & 2010 & $\begin{array}{c}\text { Asian Pacific } \\
\text { Journal of cancer } \\
\text { prevention }\end{array}$ \\
\hline 02 & $\begin{array}{l}\text { Feasibility of Quality of life assessment in routine clinical } \\
\text { oncology practice: A Tunisian Study }{ }^{15} \text {. }\end{array}$ & $\begin{array}{l}\text { A. Masmoudi } \\
\text { M. Frikha } \\
\text { J. Daoud }\end{array}$ & 2009 & $\begin{array}{l}\text { Earnest Meditterra- } \\
\text { neah Journal }\end{array}$ \\
\hline 03 & $\begin{array}{l}\text { The effect of education in managing side effect in women } \\
\text { receiving chemoterapy for treatment of breast câncer }{ }^{16} \text {. }\end{array}$ & $\begin{array}{l}\text { Susan A. Williams } \\
\text { M. Schreier }\end{array}$ & 2004 & $\begin{array}{l}\text { Oncology Nursing } \\
\text { Forum }\end{array}$ \\
\hline
\end{tabular}

QUADRO 2 - Síntese dos estudos sobre educação em saúde e quimioterapia contra câncer de mama, 2018.

\begin{tabular}{|c|c|c|c|c|}
\hline ID & OBJETIVO & $\begin{array}{l}\text { MODALIDADE DA } \\
\text { PESQUISA }\end{array}$ & $\begin{array}{l}\text { NÍVEL DE } \\
\text { EVIDÊNCIA }\end{array}$ & SÍNTESE DOS RESULTADOS \\
\hline 01 & $\begin{array}{l}\text { Avaliar a implementação de } \\
\text { um programa de autocuidado } \\
\text { para mulheres com câncer de } \\
\text { mama. }\end{array}$ & $\begin{array}{l}\text { Exploratória na } \\
\text { modalidade de pesquisa } \\
\text { ação. }\end{array}$ & VI & $\begin{array}{l}\text { Observou-se a partir da aplicação de } \\
\text { um pré e pós teste com as participantes } \\
\text { do programa de autocuidado que houve } \\
\text { redução nos efeitos adversos para } \\
\text { quimioterapia especialmente ansiedade, } \\
\text { fadiga, depressão e insônia. }\end{array}$ \\
\hline 02 & $\begin{array}{l}\text { Avaliar a viabilidade da } \\
\text { avaliação da qualidade de } \\
\text { vida (QV) em um coorte de } \\
\text { mulheres com câncer de } \\
\text { mama em quimioterapia } \\
\text { adjuvante. }\end{array}$ & $\begin{array}{l}\text { Exploratório do tipo } \\
\text { coorte. }\end{array}$ & IV & $\begin{array}{l}\text { Foi evidenciado que a aplicação do } \\
\text { questionário específico para avaliação } \\
\text { da QV com mulheres com câncer de } \\
\text { mama (QLQ-C30) apresentava resultados } \\
\text { limitados, especialmente por não conseguir } \\
\text { avaliar condições relacionadas a imagem } \\
\text { corporal, natureza pessoal e sexualidade } \\
\text { entre as participantes. Essa limitação } \\
\text { de fatores se deu em virtude da religião } \\
\text { Islâmica e nível de instrução das mulheres } \\
\text { para realização dos relatos. }\end{array}$ \\
\hline 03 & $\begin{array}{l}\text { Determinar a eficiência de } \\
\text { fitas de aúdio informativas } \\
\text { sobre autocuidado sobre } \\
\text { efeitos colaterais da } \\
\text { quimioterapia entre pacientes } \\
\text { com câncer de mama. }\end{array}$ & $\begin{array}{l}\text { Experimental do tipo caso } \\
\text { controle. }\end{array}$ & IV & $\begin{array}{l}\text { Foram constatados resultados positivos } \\
\text { no autocuidado para as reações adversas } \\
\text { em pacientes que realizavam a escuta das } \\
\text { fitas } 30 \text { minutos antes e em momentos } \\
\text { após a sessão de quimioterapia. Houve } \\
\text { redução nos sintomas de insônia, fadiga, } \\
\text { nausea, vômito e alterações no paladar } \\
\text { em mulheres que realizaram escuta das } \\
\text { fitas duas ou mais vezes. Foi evidenciado, } \\
\text { ainda, que o ambiente de adminstração de } \\
\text { quimioterápicos não é ideal para atividades } \\
\text { educativas por expor a mulher situação de } \\
\text { estresse e aumento da ansiedade. }\end{array}$ \\
\hline
\end{tabular}


dida nos espaços coletivos a fim da realização de um diagnóstico precoce e inserção da mulher de forma imediata ao tratamento. A quimioterapia representa uma das formas de maior possibilidade de redução das complicações advindas da doença bem como de garantir um melhor prognóstico e maior sobrevida as pacientes ${ }^{17}$. Contudo, por ser uma forma de tratamento sistêmico, atinge indiscriminadamente todas as células do organismo, principalmente as de rápida proliferação, produzindo efeitos adversos, os quais estão diretamente relacionados ao estado da paciente, estadiamento da doença e das drogas usadas ${ }^{18}$.

O impacto desses efeitos no tratamento e no cotidiano das mulheres durante são tão nocivos que podem determinar a suspensão das sessões ou até mesmo levar à morte da paciente. Para que o tratamento ocorra da melhor forma possível, é necessário que a paciente esteja consciente dos efeitos adversos da quimioterapia, conhecendo as maneiras de detectá-los, preveni-los e amenizá-los $^{19}$. Na redução desses impactos e consequência a manutenção do tratamento antineoplásico tornase responsabilidade da equipe de Enfermagem orientar a paciente para seu tratamento integral, provendo-a de habilidades para que esteja ativa e consciente ao longo desse processo de cuidado.

Os estudos analisados ${ }^{14,15,16}$ pontuam, em sua totalidade, a importância da ação do profissional de enfermagem na realização de ações de educação em saúde durante o tratamento quimioterápico. Essas ações devem ocorrer de forma continuada e buscando estratégias que minimizem o abandono e potencializem as ações de autocuidado e comportamentos de busca de saúde pela mulher. Em estudo aplicando atividades educativas a pacientes em tratamento quimioterápico a partir da consulta de enfermagem observou-se que as atividades possibilitavam o desenvolvimento de uma atenção com base na visão humanística e que garantiam um atendimento do individuo em sua totalidade. Inferiu-se, ainda, que a importância da educação realizada pelo enfermeiro, durante as sessões de quimioterapia, garante medidas de prevenção de complicações mais eficazes reduzindo a terapêutica intervencionista e os tratamentos desnecessários a paciente ${ }^{20}$.

Outro aspecto relevante trabalhado nas publicações é o das metodologias de ensino/intervenção adotadas pelos profissionais e a adesão das pacientes nas ações propostas. Nestas fica evidenciado que as estratégias adotadas pela enfermagem devem ser variadas de forma a garantir atração da mulher para participação, despertando seu interesse, bem como não devem ser realizadas no ambiente de infusão de quimioterápicos por gerarem estresse e ansiedade a paciente.

A educação em saúde pode ser mediada por tecnologias que ajudem o indivíduo e família a adotar ou a modificar condutas que permitam um estado saudável, possibilitando ao profissional diversas estratégias de promoção da saúde ${ }^{19}$. Em estudo envolvendo familiares de pacientes com diagnóstico de câncer de mama ${ }^{21}$ foi evidenciada que a participação da família nas atividades educativas para reabilitação promove uma oportunidade para conhecimento da doença e reorganização da vida não somente da mulher acometida pela doença, mas também dos integrantes da rede sociofamiliar. No tocante ao desenvolvimento de estratégias de intervenção para educação em saúde desenvolvidas pela enfermagem estudos referenciam a utilização de vídeos educativos a cerca dos procedimentos e intervenções durante o tratamento, que garantiram melhor adaptação dos pacientes a quimioterapia bem como oportunizaram medidas para atender as diferentes necessidades destes promovendo fortalecimento de sua autonomia e colaborando para que o mesmo tenha participação ativa no processo saúde-doença ${ }^{22}$. Outra ferramenta utilizada como meio de fortalecimento das ações de educação do paciente em quimioterapia é o telemonitoramento que surge como um amplificador do vínculo do paciente com a equipe. O contato realizado ao longo dos intervalos, entre as datas de infusão e/ou consultas de rotina, permite que o acompanhamento atinja outra dimensão, as pacientes são avaliadas enquanto os sintomas estão mais presentes. Justamente no momento em que o tratamento quimioterápico se mostra mais árduo, o cuidado de enfermagem e o acompanhamento tornam-se imprescindíveis. Assim, o contato telefônico surge como ferramenta potencial para o cuidado integral, um instrumento eficaz para o restabelecimento da mulher ${ }^{23}$.

O uso de manuais educativos como forma de respaldar as informações ofertadas às pacientes durante a quimioterapia é citada em um dos estudos como boa estratégia, existindo como barreira para sua utilização - baixo acesso a educação nos países estudados o que não torna essa estratégia com alto impacto nessa população. Estudo implementado no Distrito Federal' ${ }^{19}$, Brasil evidenciou-se que a utilização de manuais informativos e telemonitoramento para fortalecimento dos comportamentos de pacientes em quimioterapia e autocuidado frente as reações adversas demonstraram alta efetividade na orientação dos familiares e pacientes relacionado a alimentação, fortalecimento do conhecimento sobre o tratamento e sentimento de melhora do padrão de saúde. Os pacientes verbalizaram ainda redução dos efeitos pós quimioterapia e redução do uso dos serviços de retaguarda para gerenciamento destas condições clínicas.

Mulheres com câncer de mama submetidas à quimioterapia muitas vezes têm que lidar com inúmeras complicações durante $\circ$ tratamento, o que pode interferir no desempenho das atividades habituais e na manutenção da qualidade de vida $^{24}$. Durante $\circ$ tratamento os principais 
efeitos ou toxicidades são hematológicos, gastrointestinais, cardiotoxicidade, hepatotoxicidades, toxicidade pulmonar, neurotoxicidade, disfunção reprodutiva, toxicidade vesical e renal, alterações metabólicas, toxicidade dermatológicas e reações alérgicas além da possiblidade de anafilaxia ${ }^{7}$.

Em um dos estudos analisados ${ }^{15}$ se investigou as complicações relacionadas ao tratamento quimioterápico e sua interferência na manutenção da Qualidade de Vida (QV) das pacientes com câncer mamário. Neste estudo evidenciou-se redução da qualidade de vida especialmente pelas alterações relacionadas a alimentação (náuseas, vômitos e perda do paladar), ansiedade e redução da capacidade de sono e repouso com consequente fadiga. $O$ estudo utilizou do telemonitoramento como estratégia de orientação das pacientes para o autocuidado e constatou, em seus resultados, que quanto maior a adesão das pacientes as medidas de educação propostas no contato telefônico, maiores foram o cumprimento das orientações de enfermagem, redução da automedicação para manejo dos sinais e sintomas pós quimioterapia e maior capacidade de autocuidado.

Em pesquisa realizada em no interior de São Paulo, Brasil demonstrou-se que a utilização da quimioterapia como tratamento para o câncer de mama em mulheres brasileiras não se associou a pior $\mathrm{QV}$, porém o estudo teve como limitação a utilização de um questionário genérico e não houve referências de associação do tratamento a realização de atividades de educação em saúde relacionadas ao tratamento ${ }^{25}$.

O processo de educação em saúde, bem como o processo educativo em si proporcionam a reflexão, baseada em estratégias que permitam mudanças de atitudes e comportamentos. O Enfermeiro, por sua vez, deve compreender a essência da educação em saúde e, desta forma, exercer o cuidado sob o aspecto de uma educação crítica e transformadora favorecendo o bem-estar e contemplando em suas ações, tanto individuais quanto coletivas, as necessidades biopsicossociais ${ }^{26,27}$.

Estudo $^{7}$ que utilizou o instrumento de avaliação da qualidade de vida associado a neoplasia mamária (QLQ
-C30) entre 13 pacientes em seguimento do tratamento obteve como resultado que os sintomas fadiga, náuseas e vômitos foram aqueles de maior impacto na qualidade de vida das pacientes. Outro aspecto avaliado no estudo é o do comprometimento da capacidade cognitiva, funcionamento físico e fadiga após completar o tratamento quimioterápico com evidências de piora na saúde geral. Nesse estudo não houve, também, relação do impacto das atividades de educação em saúde na manutenção da QV e autocuidado das mulheres em tratamento.

\section{CONCLUSÃO}

A análise dos manuscritos proporcionou reflexão ativa sobre a importância das ações de educação em saúde no tratamento quimioterápico para câncer mamário por evidenciar que sua implementação promove impactos desde as ações relacionadas ao bem-estar, qualidade de vida, manutenção da saúde e prevenção de complicações. O profissional de Enfermagem é sujeito ativo das ações e precisa de maior embasamento e abertura nos serviços de administração desta terapia para implementação destas ações. As evidências demonstram que quando esses ideais são aplicados no desenvolvimento de ações voltadas a pacientes em quimioterapia observa-se alto potencial de impacto na redução de efeitos adversos que são determinantes para sua sobrevida.

Em sua totalidade os estudos são de publicações internacionais e demonstram que no Brasil há a necessidade de maior investigação com níveis de evidência fortes para que ações de baixo custo e de fácil operacionalização se transformem em alto impacto frente a redução de complicações e até mortalidade entre essas pacientes.

O estudo apresenta como limitação a quantidade de fontes atuais na literatura acerca desta temática o que nos leva a indicar a realização de pesquisas com metodologias de maior evidência na área e que demonstrem resultados mais específicos no fortalecimento do autocuidado, gerenciamento dos sinais e sintomas pós quimioterapia e na manutenção da qualidade de vida das pacientes sob tratamento. 


\section{REFERÊNCIAS}

I- INSTITUTO NACIONAL DO CÂNCER - INCA. Controle do Câncer de Mama - Conceito e Magnitude. [serial online]. Avaible from: http://www2.inca.gov.br/wps/wcm/connect/ acoes_programas/site/home/nobrasil/programa_controle_ cancer_mama/conceito_magnitude. cited: 24 may 2018.

2- INSTITUTO NACIONAL DO CÂNCER - INCA. Estimativa 2017: incidência de câncer no Brasil / Instituto Nacional de Câncer José Alencar Gomes da Silva. [serial online]. Rio de Janeiro: INCA, 20I6.Avaible from: http://www.inca.gov.br/ estimativa/20I7/estimativa-2017-vI I.pdf. cited: 24 may 2018.

3- MINISTÉRIO DA SAÚDE. Secretaria de Atenção à Saúde. Departamento de Atenção Básica. Controle dos cânceres do colo do útero e da mama / Ministério da Saúde, Secretaria de Atenção à Saúde, Departamento de Atenção Básica. 2. ed. Brasília: Editora do Ministério da Saúde, 2013.

4- Costa WB et al. Mulheres com Câncer de Mama: Interações e percepções sobre o cuidado do Enfermeiro. Rev. Min. Enferm [Internet]. 2012 [acesso em 20 mai 20I8];16(I): 3137. Disponivel em: http://reme.org.br/artigo/detalhes/497

5- MG dos SANTOS, Fuly PSC.Visita domiciliar e Educação em Saúde, promovendo qualidade de vida em pacientes oncológicos. Rev enferm UFPE on line [Internet].2014 [acesso em I8 mai 2018]; 8(4):904-909. Disponível em: https:// periodicos.ufpe.br/revistas/revistaenfermagem/article/ view/9759/9883.

6- Rodrigues FSS, Polidori MM. Enfrentamento e resiliência de pacientes em tratamento quimioterápico e seus familiares. Revista brasileira de cancerologia [Internet]. 2012 [acesso em 19 mai 2018]; 58 (4): 619-627. Disponível em: http:// wwwl.inca.gov.br/rbc/n_58/v04/pdf/07-artigo-enfrentamento-resiliencia-pacientes-tratamento-quimioterapicofamiliares.pdf

7- Machado SM, Sawada NO. Avaliação da qualidade de vida de pacientes oncológicos em tratamento quimioterápico adjuvante.Texto Contexto Enferm, [Internet]. 2008 [acesso em 19 mai 2018]; 17(4): 750-7. Disponível em: http://www. redalyc.org/pdf/7 |4/7|4| | 2400|6.pdf

8- Cunha FF,Vasconcelos EV, Silva SED, Freitas K de O. Representações de pacientes oncológicos sobre o tratamento de quimioterapia antineoplásica. Rev Cuidado é fundamental [Internet]. 2017 [acesso em 20 mai 20I8]; 9 (3): 840-847. Disponível em: http://www.seer.unirio.br/index.php/cuidadofundamental/article/view/5579/pdf_I

9- Vidal AKL.Verificação do conhecimento da população pernambucana acerca do câncer de boca e dos fatores de risco - Brasil. Pesq bras odontopediatria clin integr. 2012; 12(3):383-87.

I0-Souza MT, Silva MD, Carvalho R de. Revisão Integrativa: $O$ que é e como fazer. Einstein [Internet]. 2010 [acesso em 18 mai 2018]; 8(I Pt I):102-6. Disponível em: http://www. scielo.br/pdf/eins/v8n l/pt_1679-4508-eins-8-I-0102

I I-Mendes KDS, Silveira RC de CP, Galvão CM. Revisão integra- tiva: método de pesquisa para incorporação de evidências na saúde e enfermagem. Texto contexto enfermagem [Internet]. 2008 [acesso em I8 mai 2018]; I7(4): 758-64. Disponível em: http://www.redalyc.org/pdf/7 I4/7 I4 I 2400 I7.pdf

I2-Pompeo DA, Rossi LA, Galvão CM. Revisão integrativa: etapa inicial do processo de validação de diagnóstico de enfermagem. Acta paul. enferm. [Internet]. 2009 [acesso em 18 mai 2018]; 22(4):434-438. Disponível em: http://www.scielo.br/scielo.php?script=sci_arttext\&pi$\mathrm{d}=\mathrm{S} 01$ 03-2 I 0020090004000 I 4

13- Dantas RFDB et al. Caracterização das lesões crônicas nos idosos atendidos na estratégia de saúde da família. Revista de enfermagem UFPE online [Internet]. 2016 [acesso em 19 mai 2018]; I (5):1943-52. Disponível em: https:// periodicos.ufpe.br/revistas/revistaenfermagem/article/ view/23330//8924

14- Loh SY, Yip CH, Packer T, Quek KF. Self management Pilot study on women with breast cancer: Lesson Learnt in Malaysia. Asian Pacific Cancer Prev.[internet]. 2010 [acesso em I6 mai 2018]; II: 1239-1299. Disponível em: https:// www.researchgate.net/profile/Siew_Yim_Loh/publication/266376238_Self_Management_Pilot_Study_on_Women with_Breast Cancer_Lessons_Learnt/links/542e0cac0cf27e39fa95fddb/Self-Management-Pilot-Study-on -Women-with-Breast-Cancer-Lessons-Learnt.pdf

15-Masmoudi A, Frikha A, Daoud J. Feasibility of Quality of life assessment in routine clinical oncology practice: A Tunisian Study. Esastern Mediterranean Journal [internet]. 2009 [acesso em 16 mai 2018]; 15(2). 362-368. Disponivel em: http://apps.who.int/iris/bitstream/handle//0665/II7647/I5_2_2009_0362_0368.pdf?sequen$\mathrm{ce}=1$ \&isAllowed $=\mathrm{y}$

16-Williams AS, Schreier AM. The effect of education in managing side effect in women receiving chemoterapy for treatment of breast cancer. Oncology Nursing fórum [internet]. 2004.3I [acesso em I6 mai 20I8]; 3I (I): I6-23. Disponível em: https://www.researchgate.net/profile/Ann_Schreier/ publication/8919757_The_Effect_of_Education_in_Managing_Side_Effects_in_Women_Receiving_ChemotheraPy_for_Treatment_of_Breast_Cancer/links/54d8d28d0cf250 I 3d03fdbcl/The-Effect-of-Education-in-Managing-Side-Effects-in-Women-Receiving-Chemotherapy-for-Treatment-of-Breast-Cancer.pdf

17-Guimarães RCR et al. Ações de enfermagem frente às reações a quimioterápicos em pacientes oncológicos. Revista de Pesquisa Cuidado é Fundamental Online [internet]. 2012 [acesso em 18 mai 2018];7(2):2440-2452. Disponível em: http://www.redalyc.org/html/5057/505750946034/.

18-Soares LC et al. A quimioterapia e seus efeitos adversos: Relato de clientes oncológicos. Cogitare Enferm [internet]. 2009 [acesso em I8 mai 2018]; 14(4):7| 4-9. Disponível em: http://www.redalyc.org/pdf/4836/483648977019.pdf

19-Cruz FOAM, et al. Implementação de manuais educativos 
na consulta de enfermagem: opinião dos pacientes submetidos à quimioterapia antineoplásica. Rev. Enfermagem UFPE online [internet]. 2017 [acesso em 19 de mai 20 I8]; II (5): 1757-62. Disponível em: https://periodicos.ufpe.br/revistas/ revistaenfermagem/article/view/23320//8903

20- Soffiatti NRT. Consulta de Enfermagem em ambulatório de quimioterapia: ênfase nas ações educativas. Cogitare Enferm [internet]. 2000 [acesso em 21 mai 20I8]; 5 (n. esp.): 69-72. Disponível em: https://revistas.ufpr.br/cogitare/article/view/44872/27296

2I-Nascimento AN do, Castro DS de, Amorim MHC, Bicudo SDS. Estratégias de Enfrentamento de familiares de muIheres acometidas por câncer de mama. Ciência cuidado e Saúde [internet]. 2011 [acesso em 21 mai 20I8]; I0 (4): 789-794. Disponível em: http://eduem.uem.br/ojs/index. php/CiencCuidSaude/article/viewFile/ /8324/pdf

22-Razera APR, Buetto LS, Lenza N de FB, Sonobe HM. Vídeo Educativo: estratégia de ensino-aprendizagem para pacientes em tratamento quimioterápico. Ciência cuidado e Saúde [internet]. 2013 [acesso em 21 mai 20I8]; I3(I): 172-177. Disponível em: https://www.researchgate.net/ profile/Luciana_Buetto2/publication/2837/2450_VIDEO_ EDUCATIVO_ESTRATEGIA_DE_ENSINO-APRENDIZAGEM_PARA_PACIENTES_EM_TRATAMENTO_QUIMIOTERAPICO/links/5643f6ac08ae9f9c I 3e3cc5d.pdf.
23- Vaz DC, Silva CRL da, Silva RCL da. Acompanhamento presencial e telefônico dos sintomas em mulheres com câncer de mama submetidas à quimioterapia. Revista de enfermagem UERJ [internet]. 2016 [acesso em 22 mai 20 I8]; 24 (5):el 5577. Disponível em: http://www.facenf.uerj.br/v24n5/v24n5a l6.pdf

24-Amorim JR, Silva IA, Shimizu IA. Avaliação da qualidade de sono em pacientes com câncer de mama em quimioterapia. Rev. Bras. Mastologia [internet] 2017 [acesso em 22 mai 2018]; 27 (I): 3-7.Disponível em: http://www.rbmastologia. com.br/wp-content/uploads/20I7/0I/MAS-v27n I_3-7.pdf

25-Conde DM, Pinto-Neto AM, Freitas Junior R, Aldrighi JM. Qualidade de vida de mulheres com Câncer de Mama. Rev. Bras Ginecol Obstet [internet]. 2006 [acesso em 23 mai 20 I8]; 28 (3): I95-204. Disponível em: http://www.scielo.br/ pdf/\%0D/rbgo/v28n3/30847.pdf

26-Maia JS, Santos MSC, Moraes MP, Maia LF dos S. Educação em saúde e qualidade de vida. Recien [internet]. 2014 [acesso em 22 mai 20I8]; 4(I I): 5-9. Disponível em: http:// recien.com.br/index.php/Recien/article/view/70/I3 I

27- Ramos SSR, Rodrigues LMS, Silva TASM, Balbino CM, Souza MMT, Silvino ZR. Conhecimentos, mitos e implicações para o cuidado de enfermagem no câncer de mama masculino. Revista Enfermagem atual [internet].2017 [acesso em 05 jul 2018]; I7(83): 67-72. Disponível em: https://revistaenfermagematual.com.br/uploads/revistas/2I/revista.pdf 